\title{
Glauber model for a small system using the anisotropic and inhomogeneous density profile of a proton
}

\author{
Suman Deb®, Golam Sarwar®, Dhananjaya Thakur, Pavish S., and Raghunath Sahoo®* \\ Discipline of Physics, School of Basic Science, Indian Institute of Technology Indore, \\ Simrol, Indore 453552, India \\ Jan-e Alam \\ Variable Energy Cyclotron Centre, I/AF, Bidhan Nagar, Kolkata-700064, India
}

(Received 21 October 2019; published 8 January 2020)

\begin{abstract}
Recent studies reveal that at high energies, collisions of a small system such as $p+p$ gives signatures similar to that widely observed in heavy ion collisions hinting toward the possibility of forming a medium with collective behavior. With this motivation, we have used the Glauber model, which is traditionally applied to heavy ion collisions, in a small system using the anisotropic and inhomogeneous density profile of a proton and found that the proposed model reproduces the charged particle multiplicity distribution of $p+p$ collisions at LHC energies very well. Collision geometric properties such as the mean impact parameter, the mean number of binary collisions $\left(\left\langle N_{\text {coll }}\right\rangle\right)$, and the mean number of participants $\left(\left\langle N_{\text {part }}\right\rangle\right)$ at different multiplicities are determined. Having estimated $\left\langle N_{\text {coll }}\right\rangle$, we have calculated the nuclear modificationlike factor $\left(R_{\mathrm{HL}}\right)$ in $p+p$ collisions. We also estimated eccentricity and elliptic flow as a function of charged particle multiplicity using the linear response to the initial geometry.
\end{abstract}

DOI: 10.1103/PhysRevD.101.014004

\section{INTRODUCTION}

Results of relativistic proton proton $(p+p)$ collisions are used as a reference or base line for interpreting various results of heavy ion collisions at relativistic energies, which are aimed at creation and characterization of phases of strongly interacting matter governed by quantum chromodynamics (QCD). At high temperature/density, a deconfined thermalized state of quarks and gluons called quark-gluon plasma (QGP) has been predicated by lattice QCD based calculations $[1,2]$. Values of the ratio of a certain observable measured in heavy ion collisions, such as the number of produced strange particles, production of $J / \psi$, to those measured in the $p+p$ collision are interpreted as a signature of the partonic medium formation in heavy ion collisions; e.g., the enhancement of the number of strange particles and suppression in the number of $J / \psi$ in collisions of the heavy ion with respect to that of $p+p$ (approximately scales by binary collisions) are taken as signatures of QGP formation in relativistic heavy

\footnotetext{
* Corresponding author.

Raghunath.Sahoo@cern.ch

jane@vecc.gov.in

Published by the American Physical Society under the terms of the Creative Commons Attribution 4.0 International license. Further distribution of this work must maintain attribution to the author(s) and the published article's title, journal citation, and DOI. Funded by SCOAP .
}

collisions [3-11]. Apart from taking values of such a ratio as confirmation for the creation of QGP in such collisions, they are also used in characterizing QGP as well as in verifying and constraining different theoretical models. For such interpretations, it is assumed that, in $p+p$ collisions, no partonic medium is formed. However, recent results show that such assumptions may not be correct for high multiplicity $p+p$ collisions [12-14]. Understanding of $p+p$ collisions is crucial for characterization of the QCD medium formed in a heavy ion collision.

The evolution of matter formed in relativistic heavy ion collisions is critically dependent on the initial conditions. The extraction of the signals of the QGP is strongly dependent on the initial conditions [15-17]. For example, the elliptic flow $\left(v_{2}\right)$ of hadrons are calculated using the transverse momentum $\left(p_{T}\right)$ spectrum of hadrons. The $p_{T}$ spectra are estimated using the hydrodynamical models. To solve the hydrodynamical equations, initial conditions and equations of state are required as inputs. Therefore, the shear viscosity of the system extracted through $v_{2}$ will be sensitive to initial conditions, i.e., any uncertainties in the initial condition will be reflected in the extracted value of shear viscosity [18].

In high energy heavy-ion collisions, the interpretation of results relies on the use of a model based on initial matter distribution resulting from the overlap of the two colliding nuclei at a given impact parameter (b). Indeed, for estimating quantities such as the following: (i) the centrality dependence 
of any observable expressed by the number of participating nucleons in the collision, $N_{\text {part }}$ (b); (ii) the number of binary nucleon-nucleon collisions, $N_{\text {coll }}(\mathrm{b})$ used to derive the nuclear modification factor $\left(R_{A A}\right)$ from the ratio of $A A$ over $p p$ spectra; (iii) the elliptic and triangular flow parameters $\left(v_{2}\right)$ and $\left(v_{3}\right)$ normalized by the eccentricity $\epsilon_{2}(\mathrm{~b})$ and triangulation $\epsilon_{3}(\mathrm{~b})$ of the overlap region; and (iv) the average surface area, $A(\mathrm{~b})$; and (v) path length, $L(\mathrm{~b})$ of the interaction region, knowing the nuclear overlap function $T_{A A}(\mathrm{~b})$ is important. And, this overlap function depends on a realistic model of the collision geometry [19].

Similar to heavy ion collisions, it is imperative to understand the initial condition of the medium formed in $p+p$ collisions for high multiplicity events. Apart from this, knowing the proper initial condition can also give a possible way to define centrality classes and the base needed for properly defining suppression factors or ratios for comparing results of event of different multiplicity classes produced in $p+p$ collisions [20]. Appropriate initial conditions can be chosen by considering that it should reproduce certain aspects of results such as multiplicity distribution or centrality distribution of various observables related to the events.

For constructing the proper initial conditions for $p+p$ collisions, at first attempt, one follows the way similar to that of heavy ion collisions. Initial conditions for heavy ion collisions are modeled in two kinds of distinct approaches: (i) one considers nucleonic or partonic collisions for energy deposition in the collision zone, and those are based on Glauber model [21-24], and (ii) QCD based calculations are employed to estimate initial energy deposition by gluonic fields originated from partonic currents of colliding nuclei [25]. So these will also be obvious approaches for modeling initial conditions in $p+p$ collisions. As models based on Glauber modeling are very successful in reproducing various results of relativistic heavy ion collisions, one can consider models for initial conditions of $p+p$ collisions which are based on a similar kind of assumptions as used for the Glauber approach used in heavy ion collisions.

The initial transverse shape of the nuclei as described by the Glauber model for heavy-ion collisions depends on a Wood-Saxon distribution, which is a two-parameter [halfdensity radius (R) and diffusivity (a)] Fermi-like distribution $(2 \mathrm{pF})$ extracted from fits to elastic lepton-nucleus data $[26,27]$, which describe the multinucleon interactions occurring in the overlap region between the colliding nuclei via a Glauber eikonal approach [28]. Whereas, in the Monte Carlo Glauber (MCG) models [29-34], event-byevent sampling of individual nucleons is done from a Wood-Saxon distribution and the average over multiple events is used to calculate properties related to collisions. Presently, an available partonic Glauber model for $p+p$ collisions does not consider the full anisotropic density profile of protons, though radial homogeneity is assumed.
In this article, we present the results of Glauber-like model calculations for $N_{\text {coll }}(\mathrm{b}), N_{\text {part }}(\mathrm{b})$ due to the quark and gluon based proton density profile, which is a realistic picture obtained by results of deep inelastic scattering that reveals the structure of the proton [21], and we used it to obtain charged particle multiplicity distribution in $p+p$ collisions at $\sqrt{s}=7 \mathrm{TeV}$. Calculated multiplicity distribution is contrasted with ALICE data, a relation of an impact parameter with multiplicity is calculated, and the multiplicity distribution of eccentricity and flow harmonics is estimated for $p+p$ collisions. In order to understand the possibility of medium formation in high-multiplicity $p+p$ collisions, we have estimated the nuclear modificationlike factor, $R_{\mathrm{HL}}$, considering low multiplicity yields as the base.

The paper is organized as follows. In the next section we discuss the formalism that is used in this work. In Sec. III, we present the results, and Sec. IV is devoted to the summary and discussions.

\section{GLAUBER FORMALISM}

In the literature, the density profiles such as hard sphere and $2 \mathrm{pF}$ functions are used traditionally to formulate the Glauber model for a heavy ion and even for protons [34]. All these profiles can also be extended to the proton model by considering the radially symmetric parton density. In fact, in the case of a proton, several density profiles have been considered to estimate the initial conditions, most of them assume an azimuthally symmetric density profile, and those are mainly different in the phenomenological parametrization of radial variations [35]. But the standard model postulates that a proton consists of three effective quarks (constituent) and gluons within it. Thus distribution of such a configuration is less likely to be radially symmetric, because we expect individual peaks in a wave function in the quarks position inside a proton indicating its presence. The necessary condition is, however, that the wave function of each effective quark and gluon should decay rapidly around the boundary of a proton (within the root mean square (RMS) area). In this regard we find only one previous work [21] to consider the azimuthally asymmetric and inhomogeneous density distribution of a proton [36,37], which is motivated by the shape of the structure function obtained in deep inelastic scattering, pointing out that multiplicity distribution produced by different models can be used to discriminate them, which can better reproduce experimental results. The differences between the present work and that reported in Ref. [21] are as follows: (i) We have considered different possible configurations of a Gaussian-fluctuating model thereby ensuring each collision as unique by assuming the initial position vectors of the three quarks to be vertices of an equilateral triangle in an $x y$ plane, and then in order to account for all possible configurations, position vectors of the quarks are parametrized by varying azimuthal and polar angles. This parametrization is done by considering tilts of the quarks' initial configuration by some angle along the 


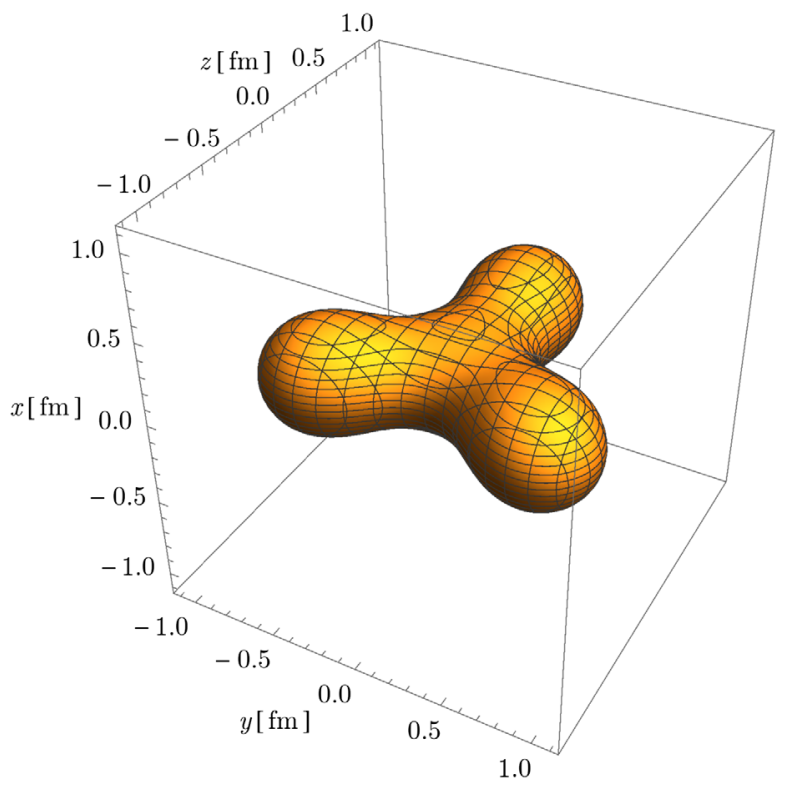

FIG. 1. Depiction of effective quarks and gluonic flux tubes connecting them within a proton [21].

$x$ axis followed by the rotation of some other angles. A similar approach is applied along the $y$ axis as well. In this process of parametrization, angles are chosen in such a way that there is no repetition of the particular configuration. (ii) For the estimation of charged particle multiplicity $\left(N_{\mathrm{ch}}\right)$, in Ref. [21], it is assumed that $N_{\mathrm{ch}}$ for each event is in a linear scaling with a number of binary collisions $\left(N_{\text {coll }}\right)$. But, in the present work, we have considered the contribution of a number of participants $\left(N_{\text {part }}\right)$ along with $\left(N_{\text {coll }}\right)$ for the estimation of charged particle multiplicity as $N_{\text {part }}$ dominates the low- $p_{T}$ region and the $N_{\text {coll }}$ contribution is higher in the high- $p_{T}$ domain. A combination of both, which is our approach, appears to be more reasonable. We have calculated elliptic flow using linear response to eccentricity.

In this study, we have used a model with fluctuating proton orientation, and it has three effective quarks and gluonic flux tubes connecting them as shown in Fig. 1. The densities of quarks $\left(\rho_{q}\right)$ and gluons $\left(\rho_{g}\right)$ are taken as the Gaussian type assuming a spherically symmetric distribution of quark densities from their respective centers and cylindrically symmetric gluon densities about the line joining two adjacent quarks as

$$
\begin{gathered}
\rho_{q}\left(\mathbf{r} ; r_{q}\right)=\frac{1}{(2 \pi)^{3 / 2} r_{q}^{3}} e^{-\frac{r^{2}}{2 r_{q}^{2}}}, \\
\rho_{g}\left(\mathbf{r} ; r_{s}, r_{l}\right)=\frac{1}{(2 \pi)^{3 / 2} r_{s}^{2} r_{l}} e^{-\frac{x^{2}+y^{2}}{2 r_{s}^{2}}-\frac{z^{2}}{2 r_{l}^{2}}},
\end{gathered}
$$

where $r_{q}$ is the radius of the quark and $r_{s}$ and $r_{l}$ are, respectively, the radius and the length of the gluon tube.
The density function under study here was taken to be [21]

$$
\begin{aligned}
& \rho_{G-f}\left(\mathbf{r} ; \mathbf{r}_{1}, \mathbf{r}_{2}, \mathbf{r}_{3}\right) \\
& =N_{g} \frac{1-\kappa}{3} \sum_{k=1}^{3} \rho_{q}\left(\mathbf{r}-\mathbf{r}_{k} ; r_{q}\right) \\
& \quad+N_{g} \frac{\kappa}{3} \sum_{k=1}^{3} \rho_{g}\left[\mathcal{R}^{-1}\left[\theta_{k}, \phi_{k}\right]\left(\mathbf{r}-\frac{\mathbf{r}_{k}}{2} ; r_{q}, \frac{r_{k}}{2}\right],\right.
\end{aligned}
$$

where $\mathcal{R}[\theta, \phi]$ transforms vector $(0,0,1)$ into $(\cos \phi \sin \theta$, $\sin \phi \cos \theta, \cos \theta)$ and $\mathbf{r}_{k}=r_{k}\left(\cos \phi_{k} \sin \theta_{k}, \sin \phi_{k} \cos \theta_{k}\right.$, $\cos \theta_{k}$ ) (where $k=1,2$, and 3 ) is the position vector of the $k$ th effective quark. $N_{g}$ is a collision energy dependent normalization factor for the density function of proton and accounts for the number of partons inside a proton. One can obtain this number by confronting the estimations to experimental observables. The free parameter $\kappa$ allows one to control the percentage of gluon body content, and here it is taken to be 0.5 as a first approximation [21]. This is the fraction of gluons (total number of gluons being $\kappa N_{g}$ ) out of all partons inside a proton at a given collision energy.

\section{A. Calculation of thickness function and overlap function}

The collision plane is taken to be in $x-y$; hence the dependence along the $z$ axis is integrated out as follows:

$$
T(x, y)=\int \rho(x, y, z) d z
$$

The calculated thickness function for the $\rho_{G-f}$ is

$$
\begin{aligned}
T(x, y)= & \sum_{k=1}^{3} \frac{N_{g}}{3} \frac{1-\kappa}{2 \pi r_{q}^{2}} e^{-l_{k}}+\frac{N_{g} \kappa}{3}\left(\frac{1}{(2 \pi)^{3 / 2} r_{s}^{2} r_{l}}\right. \\
& \left.\times \sqrt{\frac{\pi}{2}}\left(\frac{\sin ^{2} \theta_{k}}{2 r_{s}^{2}}+\frac{\cos ^{2} \theta_{k}}{2 r_{l}^{2}}\right)^{-1 / 2}\right) e^{-a_{k}\left(x-\frac{x_{k}}{2}\right)^{2}} \\
& \times e^{-b_{k}\left(y-\frac{y_{k}}{2}\right)^{2}} e^{-c_{k}\left(x-\frac{x_{k}}{2}\right)\left(y-\frac{y_{k}}{2}\right)}
\end{aligned}
$$

where $r_{s}=r_{q}$ and $r_{l}=\frac{r_{k}}{2}$; for the present studies, we have taken $r_{q}=r_{s}=0.25 \mathrm{fm}$ following Ref. [21],

$$
l_{k}=\frac{\left(x-x_{k}\right)^{2}+\left(y-y_{k}\right)^{2}}{2 r_{q}^{2}}
$$

and

$$
\begin{aligned}
a_{k}= & -\cos ^{2} \phi_{k} P_{k}+\left[\frac{1}{2 r_{s}^{2}}\left(\sin ^{2} \phi_{k}+\cos ^{2} \phi_{k} \cos ^{2} \theta_{k}\right)\right. \\
& \left.+\frac{1}{2 r_{l}^{2}}\left(\cos ^{2} \phi_{k} \sin ^{2} \theta_{k}\right)\right],
\end{aligned}
$$




$$
\begin{array}{r}
b_{k}=-\sin ^{2} \phi_{k} P_{k}+\left[\frac{1}{2 r_{s}^{2}}\left(\cos ^{2} \phi_{k}+\sin ^{2} \phi_{k} \cos ^{2} \theta_{k}\right)\right. \\
\left.+\frac{1}{2 r_{l}^{2}}\left(\sin ^{2} \phi_{k}+\sin ^{2} \theta_{k}\right)\right], \\
c_{k}=-\sin ^{2} \phi_{k} P_{k}\left[1-2\left[\frac{\tan ^{2} \theta_{k}}{r_{s}^{2}}+\frac{1}{r_{l}^{2}}\right]\right],
\end{array}
$$

and

$$
P_{k}=\frac{r_{l}^{2}-r_{s}^{2}}{4\left(\frac{r_{l}^{2}}{\cos ^{2} \theta_{k}}+\frac{r_{s}^{2}}{\sin ^{2} \theta_{k}}\right)} .
$$

The overlap function $T_{p p}(b)$ for projectile proton (A) and target proton $(\mathbf{B})$ is defined as

$T_{p p}(b)=\iint T_{A}\left(x-\frac{b}{2}, y\right) T_{B}\left(x+\frac{b}{2}, y\right) d x d y$.

Here $T_{p p}$ is sum of four components, namely quark-quark, quark-gluon, gluon-quark, gluon-gluon. Primed (unprimed) indices indicate variables corresponding to $\mathbf{B}(\mathbf{A})$. In the following, we provide an overlap function for all the possible combinations of partons.

\section{The quark-quark term}

The overlap function for the interaction of two quarks:

$$
\begin{aligned}
\left(T_{p p}\right)_{q q}(b)= & \frac{N_{g}^{2}(1-\kappa)^{2}}{36 \pi r_{q}^{2}} \sum_{k, k^{\prime}=1}^{3} \\
& \left.\times \exp \left[-\frac{\left(b-x_{k}-x_{k^{\prime}}^{\prime}\right)^{2}-\left(y_{k}-y_{k^{\prime}}^{\prime}\right)^{2}}{4 r_{q}^{2}}\right)\right] .
\end{aligned}
$$

\section{The gluon-gluon term}

The overlap function for the interaction of two gluon tubes:

$$
\left(T_{p p}\right)_{g g}(b)=\sum_{k, k^{\prime}=1}^{3} C_{k, k^{\prime}} \sqrt{\frac{\pi}{\lambda_{k, k^{\prime}}}} e^{-\frac{r_{k, k^{2}}^{2}}{4 k_{k, k^{\prime}}},}
$$

where

$$
\begin{aligned}
\gamma_{k, k^{\prime}}= & \frac{c_{k}+c_{k^{\prime}}^{\prime}}{4\left(b_{k}+b_{k^{\prime}}^{\prime}\right)}\left[c_{k}\left(b+x_{k}\right)-c_{k^{\prime}}^{\prime}\left(b-x_{k^{\prime}}\right)\right. \\
+ & \left.2\left(b_{k} y_{k}-b_{k^{\prime}}^{\prime} y_{k^{\prime}}^{\prime}\right)\right] \\
& \lambda_{k, k^{\prime}}=\left(a_{k}+a_{k^{\prime}}^{\prime}\right)-\frac{c_{k}+c_{k^{\prime}}^{\prime}}{4\left(b_{k}+b_{k^{\prime}}^{\prime}\right)}
\end{aligned}
$$

$$
\begin{aligned}
C_{k, k^{\prime}}= & A_{k} A_{k^{\prime}}^{\prime} \sqrt{\frac{\pi}{b_{k}+b_{k^{\prime}}^{\prime}}} \\
& \times \exp \left[\frac{\left[\frac{1}{2}\left[c_{k}\left(b+x_{k}\right)-c_{k^{\prime}}^{\prime}\left(b-x_{k^{\prime}}^{\prime}\right)\right]+\left(b_{k} y_{k}+b_{k^{\prime}}^{\prime} y_{k^{\prime}}^{\prime}\right)\right]^{2}}{4\left(b_{k}+b_{k^{\prime}}^{\prime}\right)}\right] \\
& \times \exp \left[-\frac{a_{k}}{4}\left(b+x_{k}\right)^{2}-\frac{a_{k^{\prime}}^{\prime}}{4}\left(b-x_{k^{\prime}}^{\prime}\right)^{2}\right. \\
& \left.-\frac{c_{k} y_{k}}{4}\left(b+x_{k}\right)+\frac{c_{k^{\prime}}^{\prime} y_{k^{\prime}}^{\prime}}{4}\left(b-x_{k^{\prime}}^{\prime}\right)\right]
\end{aligned}
$$

$$
A_{k}=\frac{N_{g} \kappa}{3} \frac{1}{(2 \pi)^{3 / 2} r_{s}^{2} r_{l}}\left(\frac{\pi}{2}\right)^{1 / 2}\left[\frac{\sin ^{2} \theta_{k}}{2 r_{s}^{2}}+\frac{\cos ^{2} \theta_{k}}{2 r_{l}^{2}}\right]^{-1 / 2} \text {. }
$$

\section{The quark-gluon term}

The overlap function for the interaction of a quark and a gluon tube:

$$
\left(T_{p p}\right)_{q g}(b)=\sum_{k, k^{\prime}=1}^{3} D_{k, k^{\prime}} \sqrt{\frac{\pi}{\alpha_{k, k^{\prime}}}} e^{\frac{\beta_{k, k^{\prime}}^{2}}{4 \alpha_{k, k^{\prime}}}}
$$

where

$$
\begin{gathered}
\alpha_{k, k^{\prime}}=\frac{1}{2 r_{q}^{2}}+a_{k^{\prime}}^{\prime}-\frac{\left(c_{k^{\prime}}^{\prime}\right)^{2}}{4\left(\frac{1}{2 r_{q}^{2}}+b_{k^{\prime}}^{\prime}\right)} \\
\beta_{k, k^{\prime}}=2 c_{k^{\prime}}^{\prime}\left[\frac{y_{k}}{r_{q}^{2}}+b_{k^{\prime}}^{\prime} y_{k^{\prime}}^{\prime}-\frac{c_{k^{\prime}}^{\prime}}{2}\left(b-x_{k^{\prime}}^{\prime}\right)-\frac{y_{k^{\prime}}^{\prime}}{4}\right] \\
-\frac{\frac{b}{2}+x_{k}}{r_{q}^{2}}+a_{k^{\prime}}^{\prime}\left(b-x_{k^{\prime}}^{\prime}\right), \\
D_{k, k^{\prime}}=E_{k, k^{\prime}} \sqrt{\frac{\pi}{2 r_{q}^{2}}+b_{k^{\prime}}^{\prime}} \exp \left[-\frac{y_{k}^{2}}{2 r_{q}^{2}}-\frac{b_{k^{\prime}}^{\prime}\left(y_{k^{\prime}}^{\prime}\right)^{2}}{4}\right] \\
\times \exp \left[-\frac{\left(\frac{b}{2}+x_{k}\right)^{2}}{2 r_{q}^{2}}-\frac{a_{k^{\prime}}^{\prime}}{4}\left(b-x_{k^{\prime}}^{\prime}\right)^{2}\right] \\
\times \exp \left[\frac{1}{4\left(\frac{1}{2 r_{q}^{2}}+b_{k^{\prime}}^{\prime}\right)}\left[\frac{y_{k}}{r_{q}^{2}}+b_{k^{\prime}}^{\prime} y_{k^{\prime}}^{\prime}-\frac{c_{k^{\prime}}^{\prime}}{2}\left(b-x_{k^{\prime}}^{\prime}\right)\right]^{2}\right] \\
\times \exp \left[\frac{c_{k^{\prime}}^{\prime} y_{k^{\prime}}^{\prime}}{4}\left(b-x_{k^{\prime}}^{\prime}\right)\right],
\end{gathered}
$$

$$
E_{k, k^{\prime}}=\frac{N_{g}^{2} \kappa(1-\kappa)}{36 \pi^{2} r_{q}^{4} r_{k^{\prime}}}\left[\frac{\sin ^{2} \theta_{k^{\prime}}^{\prime}}{2 r_{q}^{2}}+\frac{2 \cos ^{2} \theta_{k^{\prime}}^{\prime}}{r_{k^{\prime}}^{2}}\right]^{-1 / 2} \text {. }
$$

\section{The gluon-quark term}

The overlap function for the interaction of a gluon tube and a quark: 


$$
\left(T_{p p}\right)_{g q}(b)=\sum_{k, k^{\prime}=1}^{3} F_{k, k^{\prime}} \sqrt{\frac{\pi}{\delta_{k, k^{\prime}}}} \exp \left[\frac{\eta_{k, k^{\prime}}^{2}}{4 \delta_{k, k^{\prime}}}\right]
$$

where

$$
\begin{gathered}
\delta_{k, k^{\prime}}=a_{k}+\frac{1}{2 r_{q}^{2}}-\frac{c_{k}^{2}}{4\left(\frac{1}{2 r_{q}^{2}}+b_{k}\right)}, \\
\eta_{k, k^{\prime}}=a_{k}\left(b+x_{k}\right)-\frac{1}{r_{q}^{2}}\left(\frac{b}{2}-x_{k^{\prime}}^{\prime}\right)-\frac{2 c_{k}}{4\left(\frac{1}{2 r_{q}^{2}}+b_{k}\right)} \\
\times\left[b_{k} y_{k}+\frac{y_{k^{\prime}}^{\prime}}{r_{q}^{2}}+\frac{c_{k}}{2}\left(b+x_{k}\right)\right], \\
F_{k, k^{\prime}}=G_{k, k^{\prime}} \sqrt{\frac{\pi}{2 r_{q}^{2}}+b_{k}} \exp \left[\frac { 1 } { 4 ( \frac { 1 } { 2 r _ { q } ^ { 2 } } + b _ { k } ) } \left[b_{k} y_{k}+\frac{y_{k^{\prime}}^{\prime}}{r_{q}^{2}}\right.\right. \\
\left.\left.+\frac{c_{k}}{2}\left(b+x_{k}\right)\right]^{2}\right] \exp \left[-\frac{c_{k} y_{k}}{4}\left(b+x_{k}\right)-\frac{y_{k^{\prime}}^{\prime}}{2 r_{q}^{2}}-\frac{b_{k} y_{k}^{2}}{4}\right] \\
\times \exp \left[-\frac{a_{k}}{4}\left(b+x_{k}\right)^{2}-\frac{1}{2 r_{q}^{2}}\left(\frac{b}{2}-x_{k^{\prime}}^{\prime}\right)^{2}\right] \\
G_{k, k^{\prime}}=\frac{N_{g}^{2} \kappa(1-\kappa)}{36 \pi^{2} r_{q}^{4} r_{k}}\left[\frac{\sin ^{2} \theta_{k}}{2 r_{q}^{2}}+\frac{2 \cos ^{2} \theta_{k}}{r_{k}^{2}}\right]^{-1 / 2}
\end{gathered}
$$

Together the total overlap function is the sum of four terms given by Eqs. (12), (13), (18), and (23),

$$
\begin{aligned}
T_{p p}(b)= & \left(T_{p p}\right)_{q q}(b)+\left(T_{p p}\right)_{g g}(b)+\left(T_{p p}\right)_{q g}(b) \\
& +\left(T_{p p}\right)_{g q}(b) .
\end{aligned}
$$

\section{B. Calculation of $N_{\text {coll }}$ and $N_{\text {part }}$}

We define the number of binary collisions $\left(N_{\text {coll }}\right)$ of partons in a $p+p$ collision at a given impact parameter (b) as follows:

$$
N_{\text {coll }}(b)=\sigma_{\text {eff }} T_{p p}(b),
$$

where $\sigma_{\text {eff }}$ is the effective partonic cross section. It should be mentioned here that quark-quark, quark-gluon, and gluon-gluon interaction cross sections will be different due to different color factors of quarks and gluons [38]. However, we use a common partonic cross section here, which is extracted from the fits to the data as in Ref. [39], which avoids limitations of theoretical calculations at the cost of losing the information regarding the difference in individual types of interactions. In the absence of experimental information and nonperturbative QCD based calculations of the individual cross section (e.g., $g g, q g$, and $q q$ processes), we have taken a common cross section for all partons as $\sigma_{\text {eff }}$. In line with the previous studies [21,40], we fix $\sigma_{\text {eff }}=4.3 \pm 0.6 \mathrm{mb}$ [39] with $N_{g}=10$ partons, so as to reproduce the experimental value of inelastic cross section, $\sigma_{p p}=60 \mathrm{mb}$ [41] for the $p+p$ collision at $\sqrt{s}=7 \mathrm{TeV}$. This accounts for the only nontrivial dependence of the Glauber calculation on the beam energy $\sqrt{s}$. Previous studies [21,39] have assumed linear scaling of charged hadron $\left(N_{\text {ch }}\right)$ multiplicity with $N_{\text {coll }}$ only. In contrast to this assumption, we have considered the dependence of $N_{\mathrm{ch}}$ on a number of participant partons $\left(N_{\text {part }}\right)$ and $N_{\text {coll }}$. Further, the relationship between $N_{\text {part }}$ and $N_{\text {coll }}$ is considered nonlinear as that of the heavy ion collisions assuming a three-dimensional shape. Thus, the number of participating partons at impact parameter $b$ is given as

$$
N_{\text {part }}(b) \propto N_{\text {coll }}^{1 / x}(b),
$$

where $x$ is a parameter.

By considering $f$ as a fraction of charged hadron multiplicity produced from binary collisions, we have a two-component model for the estimation of a number of charged particles given as

$$
\frac{d N_{\mathrm{ch}}}{d \eta}=n_{p p}\left[(1-f) \frac{N_{\mathrm{part}}}{2}+f N_{\mathrm{coll}}\right],
$$

where $n_{p p}$ is a constant of proportionality, which represents the charged particle multiplicity density in pseudorapidity for $p+p$ collisions, and $f$ is a free parameter.

\section{RESULTS}

Assuming initial position vectors of three quarks to be vertices of the equilateral triangle in the $x y$ plane as $\mathbf{r}_{1}=\left(\frac{d}{4}, \frac{\sqrt{3}}{4} d, 0\right), \mathbf{r}_{2}=\left(\frac{d}{4},-\frac{\sqrt{3}}{4} d, 0\right)$, and $\mathbf{r}_{3}=\left(-\frac{d}{2}, 0,0\right)$, where $d$ is the free parameter of the model which ensures that the length of the gluon tubes connecting quarks are fixed, i.e., $\left.\left(\left|\mathbf{r}_{1}\right|^{2}=\left|\mathbf{r}_{2}\right|^{2}=\left|\mathbf{r}_{3}\right|^{2}\right)=\frac{d^{2}}{4}\right)$. For the present study, we have taken $d=1.5 \mathrm{fm}$ [21]. Now, in order to account for all possible configurations, position vectors of quarks are parametrized by varying azimuthal and polar angles. The generalized configurations considering the tilt by $\psi$ along the $x$ axis and the rotation by the angle $\alpha$ $\mathbf{r}_{1}=\left(\frac{d}{2} \cos \left(\frac{\pi}{3}+\psi\right), \frac{d}{2} \sin \left(\frac{\pi}{3}+\psi\right) \cos \alpha,-\frac{d}{2} \sin \left(\frac{\pi}{3}+\psi\right) \sin \alpha\right)$, $\mathbf{r}_{2}=\left(\frac{d}{2} \cos \left(\frac{5 \pi}{3}+\psi\right), \frac{d}{2} \sin \left(\frac{5 \pi}{3}+\psi\right) \cos \alpha,-\frac{d}{2}\left(\sin \frac{\pi}{3}+\psi\right) \sin \alpha\right)$, and $\mathbf{r}_{3}=\left(\frac{d}{2} \cos (\psi), \frac{d}{2} \sin \psi \cos \alpha,-\frac{d}{2} \sin \psi \sin \alpha\right)$, and considering the tilt by $\gamma$ along the $y$ axis and the rotation by the angle $\beta$ are $\mathbf{r}_{1}=\left(\frac{d}{2} \cos \left(\frac{\pi}{3}+\gamma\right) \cos \beta, \frac{d}{2} \sin \left(\frac{\pi}{3}+\gamma\right)\right.$, $\left.\frac{d}{2} \cos \left(\frac{\pi}{3}+\gamma\right) \sin \beta\right), \quad \mathbf{r}_{2}=\left(\frac{d}{2} \cos \left(\frac{5 \pi}{3}+\gamma\right) \cos \beta, \frac{d}{2} \sin \left(\frac{5 \pi}{3}+\gamma\right)\right.$, $\left.-\frac{d}{2} \cos \left(\frac{\pi}{3}+\gamma\right) \sin \beta\right), \quad$ and $\quad \mathbf{r}_{3}=\left(\frac{d}{2} \cos (\gamma) \cos \beta, \frac{d}{2} \sin \gamma\right.$, $\left.\frac{d}{2} \cos \gamma \sin \beta\right)$.

In the above configurations, $\psi$ and $\gamma \epsilon\left(0, \frac{2 \pi}{3}\right), \alpha \epsilon(0, \pi)$, and $\beta \epsilon(0,2 \pi)$. In our present study, we have taken $x$ in Eq. (30) to be 0.75 as $N_{\text {coll }}$ scales as $A^{4 / 3}$ for similar target and projectile nuclei with mass numbers $A$ for heavy ion collisions and are spherical in shape. In our work, this 


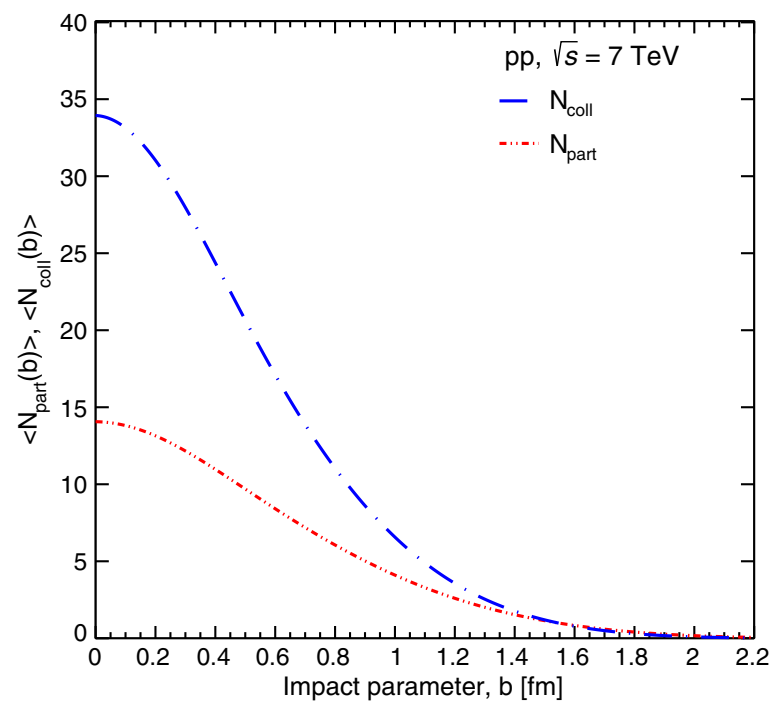

FIG. 2. $\quad N_{\text {coll }}$ and $N_{\text {part }}$ as a function of impact parameter (b) for different numbers of terms contractions.

consideration of $x=0.75$ holds well because when the plane formed by connecting centers of each quark is randomly rotated as part of the Monte Carlo simulation for accounting all possible configurations of collision geometry, the overall angular space is exhausted, thus making collision geometry to be a closely spherical overlap with preserving contributions from each of the different configurations; hence the factor of 0.75 is taken so that it accounts for general spherical overlap in heavy-ion collisions. We have also chosen the RMS radius of proton and quark as $1 \mathrm{fm}$ and $0.25 \mathrm{fm}$, respectively.

\section{A. Number of binary collisions and participants as a function of impact parameter}

We have used Eqs. (29) and (30), to estimate $N_{\text {coll }}$ and $N_{\text {part }}$. Figure 2 shows the mean value of $N_{\text {coll }}$ (upper curve) and $N_{\text {part }}$ (lower curve) as a function of impact parameter $(b)$. Toward higher values of $b$, the difference between the two curves effectively vanishes. Similar trends were observed for $\mathrm{Au}+\mathrm{Au}$ and $\mathrm{Cu}+\mathrm{Cu}$ collisions at $\sqrt{s_{N N}}=200 \mathrm{TeV}[19]$.

\section{B. Charged particle multiplicity estimation}

Two-component models have been used in heavy-ion phenomenology for a long time to estimate the chargedparticle multiplicity $[42,43]$. The inelastic cross section, $\sigma_{N N}^{\text {inel }}$, which depends on collision energy, is used as input for the MC Glauber model. In our current study, we have used a similar approach for $p+p$ collisions as well, where nucleons are replaced by partons (quarks and gluons) and

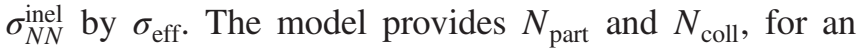
event with a given impact parameter and collision energy which is discussed in the previous section. As in heavyion collisions, the concept of "ancestors" (independently

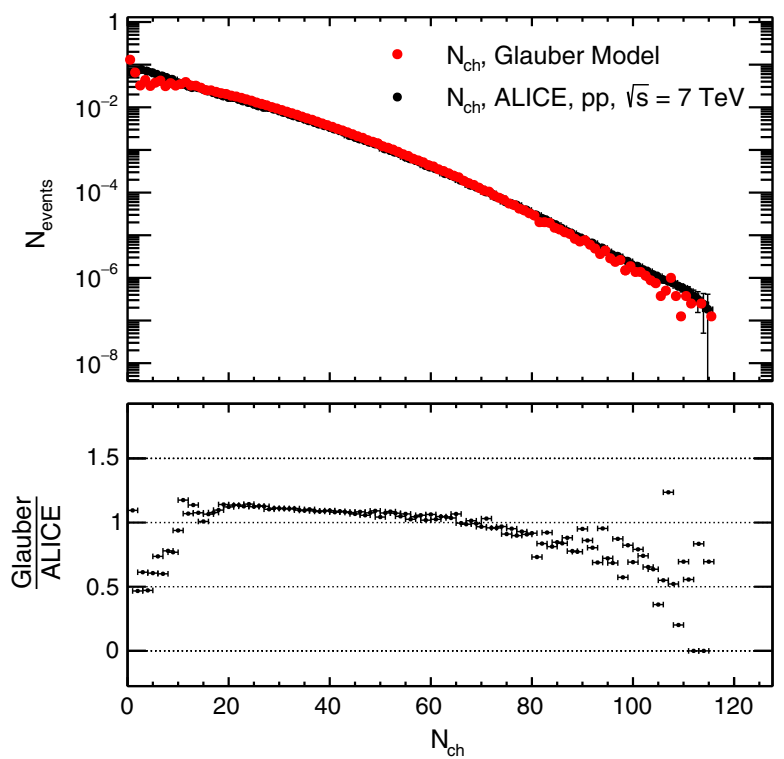

FIG. 3. Upper panel: Comparison of charged multiplicity distribution obtained from present work and ALICE experiment for $p+p$ collisions at $\sqrt{s}=7 \mathrm{TeV}$ [45]. Black dots represents ALICE data and red dots represent present work. Lower panel: Ratio of this work to the ALICE experimental data.

emitting sources of particles) has been introduced for a given value of $N_{\text {part }}$ and $N_{\text {coll }}$. The number of ancestors can be parametrized by a two-component model given by $[42,43]$

$$
N_{\text {ancestors }}=f N_{\text {part }}+(1-f) N_{\text {coll }} .
$$

The two-component model divides the parton-parton collisions into soft and hard interactions: the multiplicity of particles produced by soft interaction is proportional to $N_{\text {part }}$ and hard interaction is proportional to $N_{\text {coll }}$. As the negative binomial distribution (NBD) is able to well reproduce the charged-particle distribution in $p+p$ collisions [44], we use the two-parameter NBD to calculate the probability of producing $n$ particles per ancestor:

$$
P(n ; \bar{n}, k)=\frac{\Gamma(n+k)}{\Gamma(k) \Gamma(n+1)}\left[\frac{\bar{n}}{k+\bar{n}}\right]^{n}\left[\frac{k}{k+\bar{n}}\right]^{k},
$$

where $\bar{n}$ is the average multiplicity and $k$ characterizes the width of the distribution. By the use of different combinations of $f$ [Eq. (32)], $\bar{n}$ and $k$ [Eq. (33)] we have repeated the process of obtaining the multiplicity distribution for a large sample of events, until our model simulates the experimental multiplicity distribution. We have also calculated the ratio of $N_{c h}$ obtained from our model to that of the experimental value and is represented in Fig. 3 for $p+p$ collisions at $\sqrt{s}=7 \mathrm{TeV}$. The best agreement for $N_{\mathrm{ch}}$ distribution obtained by our model with experimental data is found for $f=0.85, \bar{n}=8$, and $k=0.13$. From Fig. 3, it 


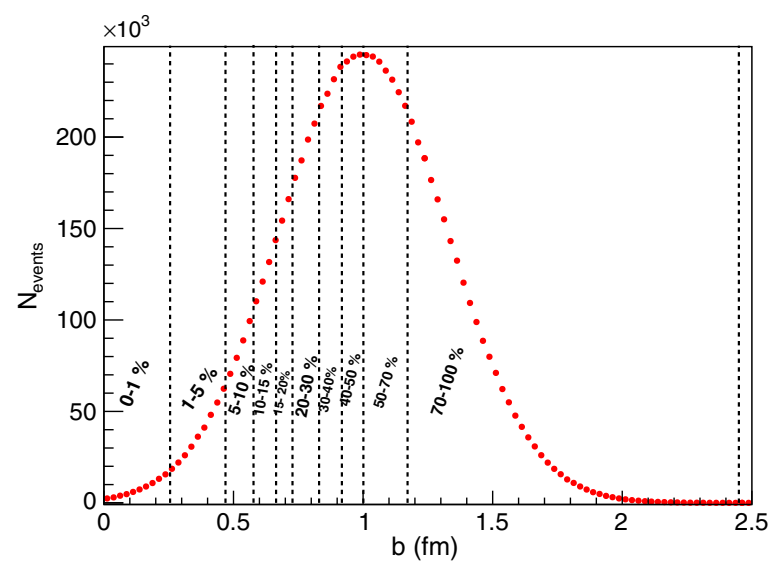

FIG. 4. Input impact parameter (b) profile for $p+p$ collisions.

can be seen that our model well describes the data in the mid multiplicity region $\left(15<N_{\mathrm{ch}}<90\right)$, with $5 \%-10 \%$ discrepancy. However, toward the low and high multiplicity it is unable to reproduce the experimental measurement. The inability of the model to explain the extreme low and high multiplicity region might be due to the lower probability of the collision impact parameter, when derived in the Monte Carlo model from a Gaussian distribution.

\section{Centrality estimation}

The centrality is usually expressed as a percentage of the total interaction cross section, $\sigma$ [46]. Impact parameter distribution is taken as input to our current model. So, the centrality percentile of a $p+p$ collision with $b$ is defined by integrating the impact parameter distribution as

$c_{1}=\frac{\int_{0}^{b 1} d N / d b d b}{\int_{0}^{\infty} d N / d b d b}, \quad c_{2}=\frac{\int_{b 1}^{b 2} d N / d b d b}{\int_{0}^{\infty} d N / d b d b}, \ldots$,

where $c_{1}, c_{2}, \ldots$, are the percentile bins and $b_{1}, b_{2}, \ldots$, are the impact parameters. More clearly, the $c_{1}$ percentage of the total number of events of impact parameter distribution fall in the interval $\left(b_{1}, b_{2}\right)$ and so on. For the current analysis, a Gaussian distribution with mean 1 and standard deviation of 0.32 has been used as an input impact parameter distribution, which is shown in Fig. 4, so that the distribution function vanishes beyond the proton radius $(\approx 2 \mathrm{fm})$.

We have also tested different forms of impact parameter distributions, but the Gaussian distribution is found to be a suitable choice to describe the charged-particle multiplicity distribution. Once, we get the ranges of the impact parameter corresponding to each centrality, we have projected it to $N_{\text {ch }}, N_{\text {part }}$, and $N_{\text {coll }}$ to calculate $\left\langle N_{\text {ch }}\right\rangle,\left\langle N_{\text {part }}\right\rangle$, and $\left\langle N_{\text {coll }}\right\rangle$ corresponding to each $b$ range. Figure 5 represents the multiplicity distribution for each percentile bin. Table I shows the value of $\left\langle N_{\text {ch }}\right\rangle,\left\langle N_{\text {part }}\right\rangle$, and $\left\langle N_{\text {coll }}\right\rangle$, obtained by using our model along with the $\left\langle N_{\mathrm{ch}}\right\rangle$ value of ALICE for $p p$ collisions at $\sqrt{s}=7 \mathrm{TeV}$.

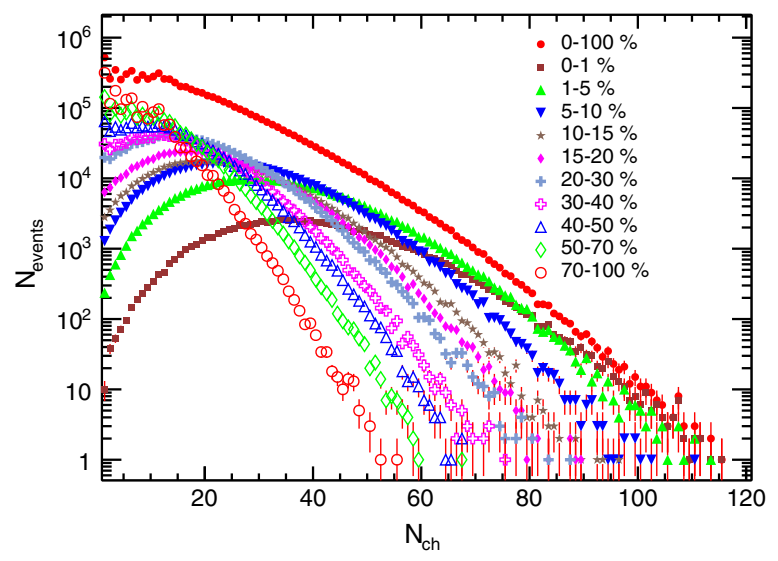

FIG. 5. Charged-particle multiplicity distribution in different percentile bins for $p+p$ collisions at $\sqrt{s}=7 \mathrm{TeV}$.

It can be clearly seen that the calculated $\left\langle d N_{\mathrm{ch}} / d \eta\right\rangle$ is well consistent with the experimental value, except for the high and low multiplicity regions. This is because of the artifact of incapability of our model to describe the charged-particle distribution in that region (Fig. 3). However, it is to be noted that the input $\sigma_{g g}^{\text {inel }}=0.43 \pm$ $0.06 \mathrm{fm}^{2}$ contains $14 \%$ uncertainty and the same amount of uncertainty (14\%) is associated with each $\left\langle d N_{\mathrm{ch}} / d \eta\right\rangle$. From our model, we found $\left\langle d N_{\mathrm{ch}} / d \eta\right\rangle=7.47$ for minimum bias $(0-100 \%)$ collisions, which is a little higher from the experimental value, $\left\langle d N_{\mathrm{ch}} / d \eta\right\rangle=6.01 \pm 0.01_{-0.12}^{+0.20} \quad$ [47]. This discrepancy needs to be understood.

\section{The ratio $\boldsymbol{R}_{H L}$ for high to low multiplicity events}

In order to understand the possibility of a formation of a medium in high-multiplicity events in $p+p$ collisions, we define a variable as

$$
R_{\mathrm{HL}}\left(p_{T}\right)=\frac{d^{2} N /\left.d p_{T} d \eta\right|^{\mathrm{HM}}}{d^{2} N /\left.d p_{T} d \eta\right|^{\mathrm{LM}}} \times \frac{\left\langle N_{\mathrm{coll}}^{\mathrm{LM}}\right\rangle}{\left\langle N_{\mathrm{coll}}^{\mathrm{HM}}\right\rangle},
$$

which is similar to the nuclear modification factor $R_{A A}$ in heavy-ion collisions. Here, $d^{2} N /\left.d \eta d p_{T}\right|^{\mathrm{HM}}, d^{2} N /$ $\left.d \eta d p_{T}\right|^{\mathrm{LM}}$, and $\left\langle N_{\text {coll }}^{\mathrm{LM}}\right\rangle\left(\left\langle N_{\text {coll }}^{\mathrm{HM}}\right\rangle\right)$ are charged particle yields in high-multiplicity, low-multiplicity $p+p$ collisions at $\sqrt{s}=7 \mathrm{TeV}$ [48], the mean number of binary collisions in low (high) multiplicity $p+p$ events, respectively. The upper panel of Fig. 6 shows the transverse momentum spectra of charged particles in high-multiplicity (VOM I), second high multiplicity (VOM II), and low multiplicity (VOM X) events obtained from Ref. [48]. And the lower panel shows the $R_{\mathrm{HL}}$ defined in Eq. (35). For such a definition of $R_{\mathrm{HL}}$, it is observed for all charged particles for $p_{T}<1 \mathrm{GeV} / c$, value of $R_{\mathrm{HL}}<1$ and for $p_{T}>1 \mathrm{GeV} / c$, it is greater than 1 . However, it tends to reduce at very high $p_{T}$. And for $p_{T}>1 \mathrm{GeV}$, the value of the factor is higher for higher multiplicities. 


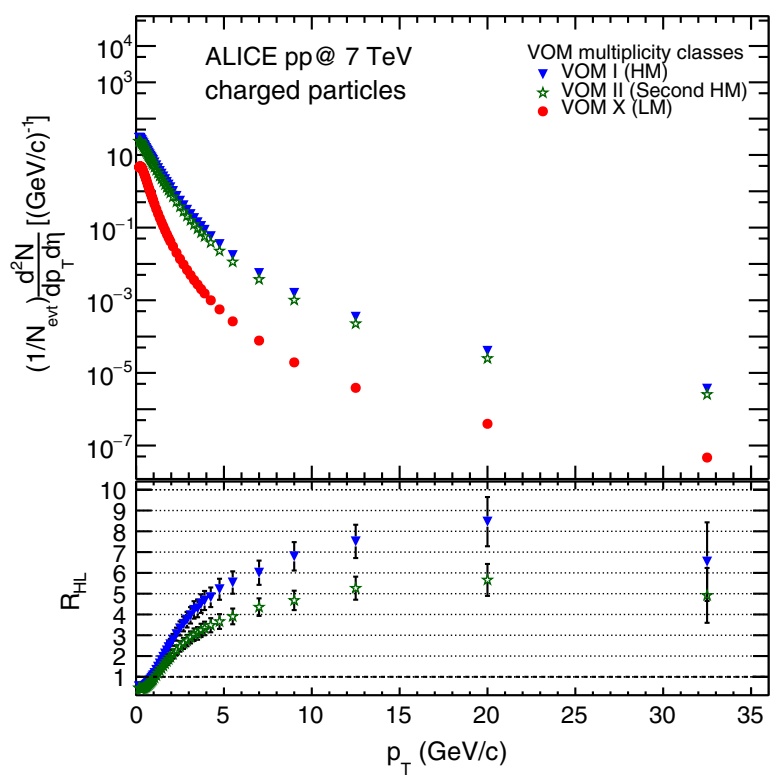

FIG. 6. Upper panel: Transverse momentum spectra of charged particles in $p+p$ collisions at $\sqrt{s}=7 \mathrm{TeV}$ [48] for VOM multiplicity classes, viz., highest (HM), second highest (second $\mathrm{HM}$ ), and lowest multiplicity (LM) class. Lower panel: $R_{\mathrm{HL}}$ obtained from the ratio of the differential yield at high multiplicity and the second highest multiplicity classes with the low multiplicity class scaled by $\left\langle N_{\text {coll }}\right\rangle$.

Figure 7 shows results of $R_{\mathrm{HL}}$ for identified particles, pion $\left(\pi^{+}+\pi^{-}\right)$, kaon $\left(K^{+}+K^{-}\right)$, proton $(p+\bar{p})$ for $p+$ $p$ collisions at $\sqrt{s}=7$. It is found that $R_{\mathrm{HL}}<1$ for a proton for $p_{T}<1 \mathrm{GeV}$, which is the same as observed in the case of charged particles. However, for pion and kaon $R_{\mathrm{HL}}<1$ for $p_{T}<0.8 \mathrm{GeV}$. It is also observed that for $p_{T}<$ $1.9 \mathrm{GeV}$, these identified particles have almost the same value of $R_{\mathrm{HL}}$, and for $p_{T}>1.9 \mathrm{GeV}$, the value is almost the same for the pion and kaon but the value for the proton is larger and increases with $p_{T}$ sharply up to $p_{T}=5 \mathrm{GeV}$, and then saturates within uncertainties. But for the pion

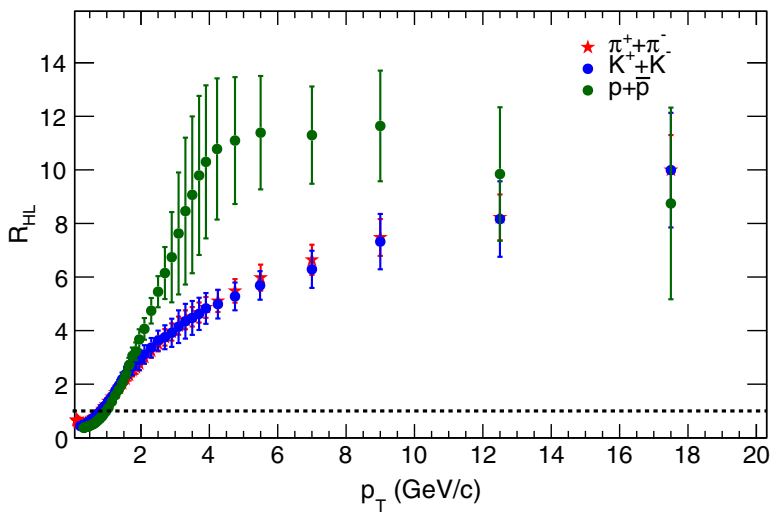

FIG. 7. Nuclear modificationlike factor obtained from Eq. (35) for the pion, kaon, and proton in $p+p$ collisions at $\sqrt{s}=$ $7 \mathrm{TeV}$ [48]. and kaon, the factor increases monotonically with a decreasing slope from $p_{T}>1.9 \mathrm{GeV}$, where the trend splits for the proton and the other two hadrons.

It is reported [49] that the proton shows a distinct behavior in this regard other than the hadrons produced in $p-\mathrm{Pb}$ collisions. Also for $p$ - $\mathrm{Pb}$ collisions, it is reported that the factor, $R_{p \mathrm{~Pb}}>1$, for all charged particle for $p_{T}>$ $2.5 \mathrm{GeV}[49,50]$. For $p-\mathrm{Pb}, R_{p \mathrm{~Pb}}$ saturates to unity for $p_{T}>2 \mathrm{GeV}$, and it is also found that for $p+p, R_{\mathrm{HL}}$ shows an almost similar trend but with a larger value of the factor with saturationlike behavior starting after $p_{T}=2 \mathrm{GeV}$. We note that the $R_{\mathrm{HL}}$ values above unity for $p_{T}>1 \mathrm{GeV}$ may be qualitatively similar to other observed enhancements due to the Cronin effect and radial flow in $p A$ and $d A$ systems [51,52], as conjectured for a similar behavior of $R_{p \mathrm{~Pb}}$ [50], where the moderate excess at high $p_{T}$ is suggestive of antishadowing effects in the nuclear parton distribution function [53].

\section{E. Estimation of elliptic flow}

For a long time, $p+p$ collisions were considered as the baseline measurements for the determination of the deconfined state of matter, i.e., QGP formed in a nuclear collision. A recent observation of $p+p$ collisions at LHC energies hints toward a collective effect; thus, it becomes imperative to review the earlier view. In this regard, we have also calculated eccentricity $(\epsilon)$ using the present approach. The asymmetry ratio between semiaxis dimensions of the overlap region weighted by $N_{\text {coll }}$ at a particular $b$ can be used to obtain $\epsilon$ as [40]

$$
\epsilon(b)=\frac{\int\left(y^{2}-x^{2}\right) n_{\mathrm{coll}}(x, y, b) d x d y}{\int\left(y^{2}+x^{2}\right) n_{\mathrm{coll}}(x, y, b) d x d y}
$$

where $n_{\text {coll }}(x, y, b)=\sigma_{g g} T_{a}\left(x-\frac{b}{2}, y\right) T_{b}\left(x+\frac{b}{2}, y\right)$ represents the impact plane binary collision density. We have calculated $\epsilon(b)$ by using Eq. (36) by considering a sum of four components, namely quark-quark, quark-gluon, gluon-quark, and gluon-gluon. Figure 8 shows the eccentricity for the $p+p$ collision at $\sqrt{s}=7 \mathrm{TeV}$ obtained using Eq. (36), and it is observed to increase with $b$ and seems to saturate toward larger $b$.

Using $\epsilon$, we have obtained the elliptic flow $\left(v_{2}\right)$ as a function of $b$ by considering the scaling: $v_{2}=\Omega \epsilon$, where $\Omega=0.3 \pm 0.02$ [39]. Although we have considered a linear scaling to understand the variation of $v_{2}$ with multiplicity, as a matter of fact $v_{2}$ should be calculated by using relativistic hydrodynamics with relevant initial conditions and the equation of state as inputs.

By geometry, $v_{2}(b)$ will follow the general trend of $\epsilon(\mathrm{b})$. It is found that the overlap of two hard spheres with infinitely sharp edges yields artificially large eccentricities [54].

In Fig. 9, we have compared our estimation of the variation of $v_{2}$ with the charged particle multiplicity for 


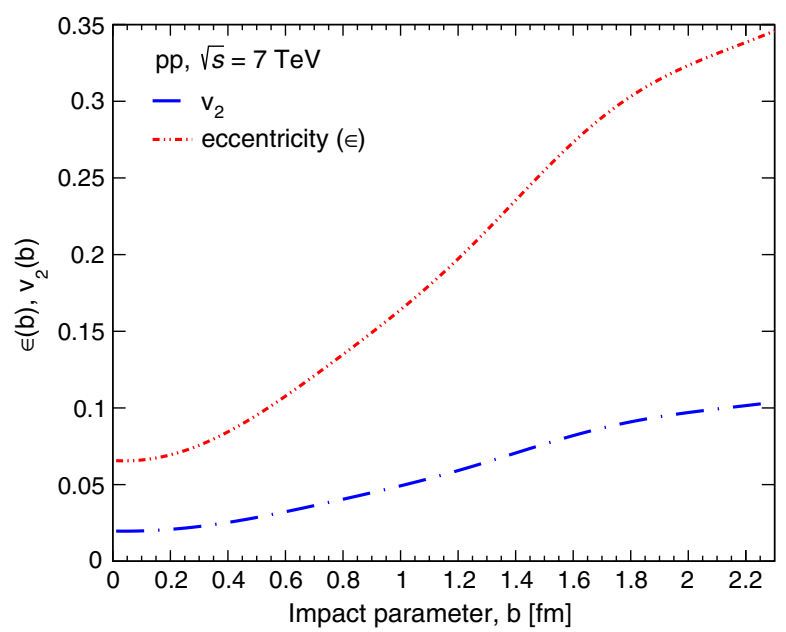

FIG. 8. Eccentricity $(\epsilon)$ and elliptic flow $\left(v_{2}\right)$ as functions of the impact parameter in $p+p$ collisions at $\sqrt{s}=7 \mathrm{TeV}$.

$p+p$ collision at $\sqrt{s}=7 \mathrm{TeV}$ with the experimental result at $\sqrt{s}=13 \mathrm{TeV}$ [55]. This is due to the fact that the data for collisions at $\sqrt{s}=13 \mathrm{TeV}$ were not available at the time of the reporting of this work to constrain our model. That does not prevent us from the comparison, since in Ref. [56], it is reported that the value of $v_{2}$ for collisions at $\sqrt{s}=2.76 \mathrm{TeV}$ and $\sqrt{s}=13 \mathrm{TeV}$ are almost the same when measured for different transverse momenta, indicating that the collision energy dependence of $v_{2}$ is weak. It is observed that for $N_{\mathrm{ch}} \gtrsim 8$, our estimation of $v_{2}$ with a linear response to initial geometry reproduces the value obtained from the experiment within the error bars. However, for lower multiplicities, our estimation with a linear response to the initial eccentricity falls short of that obtained from experimental data. This may be due to effects other than the collective linear response or the final state effects. Though the charged particle multiplicity variation of $v_{2}$ for $p+p$ collisions at $\sqrt{s}=7 \mathrm{TeV}$ is not available, the elliptic flow coefficient extracted from the CMS Collaboration data at

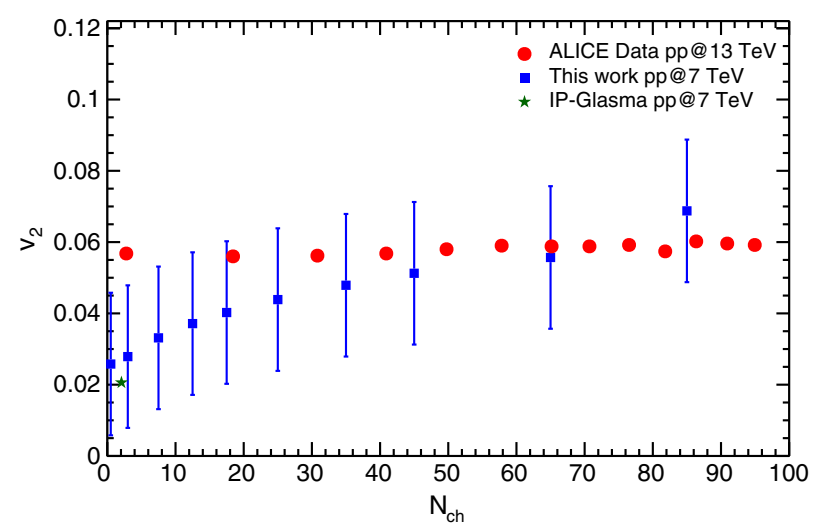

FIG. 9. Elliptic-flow $v_{2}$ as a function of multiplicity in $p+p$ collisions at LHC energies. $\sqrt{s}=7 \mathrm{TeV}$ is $0.04-0.08$ [57] and our estimation of $v_{2}$ falls within this range. We also note that this model gives $v_{2}$ similar to that of the IP-Glasma model as presented in Ref. [58] for low multiplicity region $(<8)$.

\section{SUMMARY AND DISCUSSION}

In this work, we have investigated predictions of the Glauber model for the initial condition for $p+p$ collisions, which considers an anisotropic and inhomogeneous proton density profile. The results have been contrasted with experimental data. This model for the density profile is inspired by the structure function obtained from deep inelastic scattering. Instead of distributing the positions of valence quarks randomly by keeping the center of mass intact, we have taken random orientations generated by random rotation around three spatial axes, where the center of three quarks form a plane and connecting gluon tubes always remain fixed in length. This prevents the overlap of two valence quarks in space and possible placement of a quark out of the proton radius, where these two can happen for the first kind of randomization with only the center of mass being fixed [21], and the condition that may bring extra complications in the randomization process for not allowing it, generating "spooky" correlations. However, the present approach, apart from avoiding such complications, will give a better handle for future investigations.

With all these considerations, we have studied multiplicity distribution, to obtain the impact parameter to multiplicity relation, multiplicity dependence of initial eccentricity, and azimuthal flow harmonics $\left(v_{2}\right)$. It is found that this model can well reproduce multiplicity distribution produced in $p+p$ events at ALICE, with the free parameter $f=0.85$. With properly constraining our model with experimental data and calibrating the range of $b$ with the multiplicity percentile, we have used the estimated $\left\langle N_{\text {coll }}\right\rangle$ to obtain a nuclear modificationlike factor $\left(R_{\mathrm{HL}}\right)$ for $p+p$ collisions. It is found that the defined factor $<1$ for $p_{T}<1 \mathrm{GeV}$, and beyond this, the factor $>1$. Moreover, it tends to reduce at very high $p_{T}$, and for $p_{T}>1 \mathrm{GeV}$, the value of the factor is higher for higher multiplicities. We have also studied $R_{\mathrm{HL}}$ for identified particles for $p+p$ collisions at $\sqrt{s}=7 \mathrm{TeV}$ and found that the trend for $R_{\mathrm{HL}}$ is similar to that observed in the $p$-Pb system but with an increased value. This behavior at higher $p_{T}$ may be due to noncollective flow effects, which needs further investigation.

The nonavailability of results from experiments which shows the variation of eccentricity and $v_{2}$ with multiplicity at $\sqrt{s}=7 \mathrm{TeV}$ prevents us from comparing our estimation with experimental data at $\sqrt{s}=7 \mathrm{TeV}$. However, we have compared our result of $v_{2}$ with that of $p+p$ collisions at $\sqrt{s}=13 \mathrm{TeV}$, as it is observed that the collision energy dependence of $v_{2}$ is weak. We found that the result of $v_{2}$ obtained from the present approach is in agreement with the result obtained in the IP-Glasma model in the lower multiplicity region. Also, it is found 
that the values of $v_{2}$ obtained from the present model for $N_{\text {ch }} \gtrsim 8$ are very close to that of the experimental data for $\sqrt{s}=13 \mathrm{TeV}$.

The elliptic flow $v_{2}$ measured through the anisotropic momentum distribution of the produced particle is generated by the hydrodynamic pressure gradient resulting from the spatial anisotropy of the system formed initially. Therefore, $v_{2}$ can be used to characterize the evolving medium, and to do that, any momentum dependence resulting from other sources has to be subtracted out. The initial conditions required to solve the hydrodynamic equations are quantities that depend on the spatial coordinate but are momentum independent. Therefore, the initial condition obtained in the present study will be relevant for studying the evolving matter formed in $p+$ $p$ collisions. The momentum dependent initial condition obtained in the IP-Glasma model (e.g., the work reported in Ref. [59]) can also be useful to study hydrodynamic evolution when the momentum dependence is integrated out.

\section{ACKNOWLEDGMENTS}

S. D., G. S., and R. S. acknowledge the financial support from ALICE Project No. SR/MF/PS-01/2014-IITI(G) of Department of Science \& Technology, Government of India. D. T. acknowledges UGC, New Delhi, Government of India for financial support. R. S. acknowledges the financial support from DAE-BRNS Project No. 58/14/ 29/2019-BRNS. Also, S. D., G. S., and P. S. gratefully acknowledge Patryk Kubiczek for discussion on geometrical models for $p+p$ collisions.

\section{APPENDIX: MULTIPLICITY VS GEOMETRIC PROPERTIES OF THE COLLISION}

Table I shows geometric properties $\left(\langle b\rangle,\left\langle N_{\mathrm{ch}}\right\rangle,\left\langle N_{\text {part }}\right\rangle\right.$, $\left.\left\langle N_{\text {Coll }}\right\rangle\right)$ of $p+p$ collisions for different multiplicity classes using the Glauber Monte Carlo calculation along with a negative binomial distribution fit to a charged particle multiplicity distribution at $\sqrt{s}=7 \mathrm{TeV}$ for the ALICE experiment at the LHC.

TABLE I. Geometric properties $\left(b, N_{\mathrm{ch}}, N_{\text {part }}, N_{\text {Coll }}\right)$ of $p+p$ collisions for different multiplicity classes using the Glauber Monte Carlo calculation along with a negative binomial distribution fit to a charged particle multiplicity distribution at $\sqrt{s}=7$ TeV for the ALICE experiment at the LHC.

\begin{tabular}{lcccrr}
\hline \hline Multiplicity [\%] & $b$ range [fm] & $\left\langle d N_{\text {ch }} / d \eta\right\rangle^{\text {Glauber }}$ & $\left\langle d N_{\text {ch }} / d \eta\right\rangle^{\text {expt }}$ & $\left\langle N_{\text {part }}\right\rangle$ & $\left\langle N_{\text {coll }}\right\rangle$ \\
\hline $0-1$ & $0-0.25534$ & 19.69 & $28.82_{-0.84}^{+0.86}$ & 13.142 & 31.156 \\
$1-5$ & $0.25535-0.46909$ & 16.24 & $20.34_{-0.58}^{+0.57}$ & 11.164 & 24.815 \\
$5-10$ & $0.46909-0.58484$ & 13.37 & $15.80_{-0.32}^{+0.34}$ & 9.244 & 19.478 \\
$10-15$ & $0.58484-0.66430$ & 11.61 & $13.07_{-0.22}^{+0.24}$ & 8.037 & 16.153 \\
$15-20$ & $0.66431-0.72766$ & 10.28 & $11.25_{-0.18}^{+0.19}$ & 7.131 & 13.818 \\
$20-30$ & $0.72767-0.83026$ & 8.94 & $9.21_{-0.14}^{+0.15}$ & 6.116 & 11.326 \\
$30-40$ & $0.83027-0.91819$ & 7.48 & $7.13_{-0.11}^{+0.12}$ & 5.268 & 9.215 \\
$40-50$ & $0.91820-1.00117$ & 6.49 & $5.65_{-0.09}^{+0.11}$ & 4.418 & 7.340 \\
$50-70$ & $1.00118-1.17163$ & 5.12 & $3.81_{-0.06}^{+0.07}$ & 3.395 & 5.208 \\
$70-100$ & $1.17164-2.54998$ & 3.66 & $1.66_{-0.04}^{+0.05}$ & 1.968 & 2.591 \\
\hline \hline
\end{tabular}

[1] A. Bazavov et al. (HotQCD Collaboration), Phys. Rev. D 90, 094503 (2014).

[2] S. Borsanyi, Z. Fodor, C. Hoelbling, S. D. Katz, S. Krieg, and K. K. Szabo, Phys. Lett. B 730, 99 (2014).

[3] M. Krzewicki (ALICE Collaboration), J. Phys. G 38, 124047 (2011).

[4] K. Aamodt et al. (ALICE Collaboration), Phys. Rev. Lett. 106, 032301 (2011).

[5] T. Hirano, P. Huovinen, and Y. Nara, Phys. Rev. C 84, 011901 (2011).

[6] I. Arsene et al. (BRAHMS Collaboration), Nucl. Phys. A757, 1 (2005).
[7] B. B. Back et al., Nucl. Phys. A757, 28 (2005).

[8] J. Adams et al. (STAR Collaboration), Nucl. Phys. A757, 102 (2005).

[9] E. V. Shuryak, Nucl. Phys. A750, 64 (2005).

[10] M. Gyulassy and L. McLerran, Nucl. Phys. A750, 30 (2005).

[11] B. Muller and J. L. Nagle, Annu. Rev. Nucl. Part. Sci. 56, 93 (2006).

[12] J. Adam et al. (ALICE Collaboration), Nat. Phys. 13, 535 (2017).

[13] B. Alver et al. (PHOBOS Collaboration), Phys. Rev. C 83, 024913 (2011). 
[14] V. Khachatryan et al. (CMS Collaboration), J. High Energy Phys. 09 (2010) 091.

[15] L. Yan, Nucl. Phys. A967, 89 (2017).

[16] K. Yagi, T. Hatsuda, and Y. Miake, Quark Gluon Plasma (Cambridge University Press, Cambridge, England, 2005).

[17] C. Y. Wong, Introduction to High-Energy Heavy Ion Collisions (World Scientific, Singapore, 1994).

[18] H. Song, S. A. Bass, U. Heinz, T. Hirano, and C. Shen, Phys. Rev. Lett. 106, 192301 (2011); 109, 139904(E) (2012).

[19] M. L. Miller, K. Reygers, S. J. Sanders, and P. Steinberg, Annu. Rev. Nucl. Part. Sci. 57, 205 (2007).

[20] J. Adam et al. (ALICE Collaboration), Phys. Rev. C 91, 064905 (2015).

[21] P. Kubiczek and S. D. Glazek, Lith. J. Phys. 55, 155 (2015).

[22] R. J. Glauber, Phys. Rev. 100, 242 (1955).

[23] R. J. Glauber, Nucl. Phys. A774, 3 (2006).

[24] A. Shor and R. S. Longacre, Phys. Lett. B 218, 100 (1989).

[25] B. Schenke, P. Tribedy, and R. Venugopalan, Phys. Rev. Lett. 108, 252301 (2012).

[26] C. W. De Jager, H. De Vries, and C. De Vries, At. Data Nucl. Data Tables 14, 479 (1974); 16, 580(E) (1975).

[27] H. De Vries, C. W. De Jager, and C. De Vries, At. Data Nucl. Data Tables 36, 495 (1987).

[28] R. J. Glauber and G. Matthiae, Nucl. Phys. B21, 135 (1970).

[29] X. N. Wang and M. Gyulassy, Phys. Rev. D 44, 3501 (1991).

[30] B. Alver, M. Baker, C. Loizides, and P. Steinberg, arXiv: 0805.4411 .

[31] C. Loizides, J. Nagle, and P. Steinberg, SoftwareX 1-2, 13 (2015).

[32] W. Broniowski, M. Rybczynski, and P. Bozek, Comput. Phys. Commun. 180, 69 (2009).

[33] M. Rybczynski, G. Stefanek, W. Broniowski, and P. Bozek, Comput. Phys. Commun. 185, 1759 (2014).

[34] C. Loizides, Phys. Rev. C 94, 024914 (2016).

[35] D. d'Enterria, G. K. Eyyubova, V. L. Korotkikh, I. P. Lokhtin, S. V. Petrushanko, L. I. Sarycheva, and A. M. Snigirev, Eur. Phys. J. C 66, 173 (2010).
[36] J. D. Bjorken, S. J. Brodsky, and A. Scharff Goldhaber, Phys. Lett. B 726, 344 (2013).

[37] S. D. Glazek, Few-Body Syst. 52, 367 (2012).

[38] B. L. Combridge, J. Kripfganz, and J. Ranft, Phys. Lett. 70B, 234 (1977).

[39] H. J. Drescher, A. Dumitru, C. Gombeaud, and J. Y. Ollitrault, Phys. Rev. C 76, 024905 (2007).

[40] S. D. Glazek and P. Kubiczek, Few-Body Syst. 57, 425 (2016).

[41] S. Chatrchyan et al. (CMS Collaboration), Phys. Lett. B 722, 5 (2013).

[42] X. N. Wang and M. Gyulassy, Phys. Rev. Lett. 86, 3496 (2001).

[43] D. Kharzeev and M. Nardi, Phys. Lett. B 507, 121 (2001).

[44] G. J. Alner et al. (UA5 Collaboration), Phys. Lett. 160B, 199 (1985).

[45] K. Aamodt et al. (ALICE Collaboration), Eur. Phys. J. C 68, 345 (2010).

[46] B. Abelev et al. (ALICE Collaboration), Phys. Rev. C 88, 044909 (2013).

[47] B. Abelev et al. (ALICE Collaboration), Phys. Lett. B 712, 165 (2012).

[48] S. Acharya et al. (ALICE Collaboration), Phys. Rev. C 99, 024906 (2019).

[49] G. Bencedi (ALICE Collaboration), arXiv:1609.05665.

[50] V. Khachatryan et al. (CMS Collaboration), J. High Energy Phys. 04 (2017) 039.

[51] A. Adare et al. (PHENIX Collaboration), Phys. Rev. C 88, 024906 (2013).

[52] V. Khachatryan et al. (CMS Collaboration), Phys. Rev. Lett. 115, 012301 (2015).

[53] M. Arneodo, Phys. Rep. 240, 301 (1994).

[54] S. A. Voloshin, A. M. Poskanzer, and R. Snellings, LandoltBornstein 23, 293 (2010).

[55] K. Gajdošová (ALICE Collaboration), Nucl. Phys. A982, 487 (2019).

[56] G. Aad et al. (ATLAS Collaboration), Phys. Rev. Lett. 116, 172301 (2016).

[57] P. Bozek, Eur. Phys. J. C 71, 1530 (2011).

[58] A. Bzdak, B. Schenke, P. Tribedy, and R. Venugopalan, Phys. Rev. C 87, 064906 (2013).

[59] B. Schenke, C. Shen, and P. Tribedy, arXiv:1908.06212. 